\title{
A study of organizational factors in occupational stress problems among workers in a polymer manufacturing factory
}

\begin{abstract}
Occupational stress is a major hazard for many workers. Increasing workloads, downsizing, overtime and shift work are just a few the many causes of stressful working condition. Moreover unhealthy work organization will not only affect the work performance but will affect the worker's health as well. With this in mind, a study was conducted to determine the prevalence of occupational stress and to identify the association between the organizational factors with stress level among factory workers. Through a cross sectional study, 91 manufacturing workers were randomly sampled and data was collected using a questionnaire, namely the Job Content Questionnaire (JCQ). The validated self-administered Malay version of JCQ was used to verify the socio-demographic background, the stress level of the respondents and the factors contributed to work stress which is organizational factors. JCQ contains four factors which were the psychological demand (job demand), decision latitude (job control), social support and job insecurity. Overall the prevalence of occupational stress among the respondent was $25.3 \%$. The result showed that there were significant associations between occupational stress and decision latitude $(\mathrm{p}<; 0.001)$; psychological job demand $(\mathrm{p}<; 0.001)$; and job insecurity $(\mathrm{p}=0.027)$. However, there was no significant association between occupational stress level and social support $(\mathrm{p}=0.433)$. This study showed that three out of four organizational factors had significant associations to occupational stress indicating unhealthy work organization. In addition as the prevalence is considered low, work stressors can be detected at an early stage. Therefore, preventive measure or intervention needs to implement accordingly to alleviate the negative consequences since the occupational stress can be considered as a major hazard for the workers.
\end{abstract}

Keyword: Occupational stress; Organizational factors; Job content questionnaire; Manufacturing 